\title{
Study of High Efficiency Membrane Pilot Test for Membrane Design Optimisation in Order to Reduce the Electrical Consumption
}

\author{
Federico Antonio León and Alejandro Ramos \\ Institute of Intelligent Systems and Numerical Applications in Engineering, University of Las Palmas de Gran Canaria, Tafira Campus \\ 35016 Las Palmas de Gran Canaria, Spain
}

Received: July 19, 2016 / Accepted: July 29, 2016 / Published: October 31, 2016.

\begin{abstract}
Regarding the studies of other authors about energy performance of a reverse osmosis desalination plant operating with variable pressure; about desalination efficiency in their different recent articles, we continued working in this way researching in a real seawater reverse osmosis plant following the items below. The objectives of this test are to get the minimum electrical consumption and energy costs for the operation of a sea water desalination plant using membranes. We need to compare the performance of the different membranes manufacturer's and to determine, under this new thermal scenario, the optimal configuration to get the water quality and quantity needs with the minimum energy consumption.
\end{abstract}

Key words: Minimum energy consumption, energetic efficiency, high efficiency membrane, energy costs.

\section{Introduction}

In May 2011, the new seawater intake construction works finished at a desalination plant in Almeria (Spain). From this moment on the water catchment was located at 35 meter depth instead of the 14 meter depth of the former intake.

The most significant outcome was a new thermal scenario, more stable in time and with lower maximum temperatures and higher minimum temperatures. At low temperature feed pressure is higher so we consume more electrical power [1-7].

\section{Results of the New Seawater Intake}

After this new seawater intake construction, temperatures are more stable; the feed pressure decreased 2-3 bars therefore the electrical consumption too. The energetic efficiency is one of the highest concerning issues since the energy prize had risen over

Corresponding author: Federico Antonio León, professor, research fields: desalination, energy and environment.
$25 \%$ in the last 10 years in Spain, and it represents over $50 \%$ of the operational costs of a desalination plant. Besides best practices in energy contracting have been applied in order to minimize the energy cost acquisition, any significant specific consumption reduction would lead to important economic savings. Therefore, the design of the optimal membrane configuration is a key factor for the energetic efficiency of the desalination plant.

The interest of the work is to compare the performance of the different membranes models, under this thermal scenario, in order to determine the main contributions applicable to all desalination plants with the most profitable configuration to get more water quality and quantity at the minimum energy consumption.

Because of the lower maximum temperatures (minimum temperatures remain the same) the pressure for water quality requirements accomplishment at high temperatures decreased. Therefore new design and operation opportunities appeared. Taking into account 


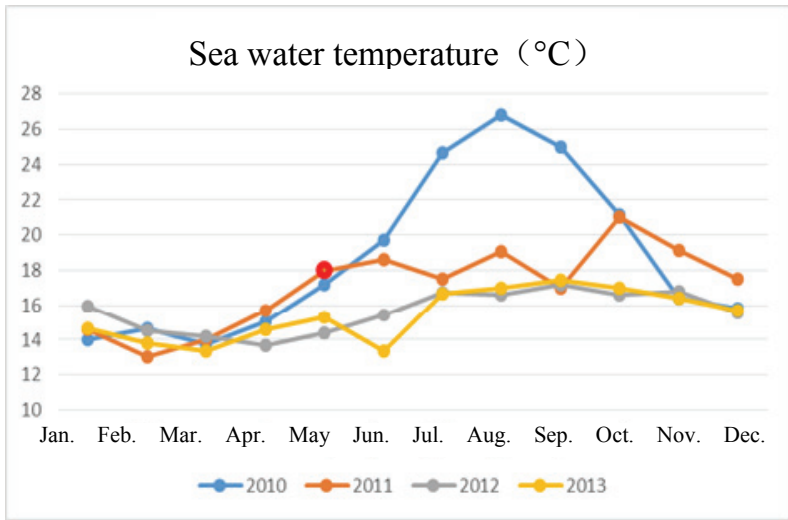

Fig. 1 Monthly raw seawater average temperatures from 2011 to 2013.

that membrane replacement and retrofit projects are about to come at this desalination plant, and under this new thermal scenario, a pilot test has been developed. Due to this pilot test we get to reduce the electrical consumption due to this high efficiency membrane test from $3.04 \mathrm{~kW} \cdot \mathrm{h} / \mathrm{m}^{3}$ to $2.60 \mathrm{~kW} \cdot \mathrm{h} / \mathrm{m}^{3}$ changing Pelton turbines to isobaric chambers. Even we are thinking to reduce it until $2.40 \mathrm{~kW} \cdot \mathrm{h} / \mathrm{m}^{3}$ introducing a Dweer equipment.

On the other hand, to determine the optimal membrane configuration and operation conditions able to achieve water quality and quantity needs under the new thermal intake conditions. The best option has to be determined by taking into account investment versus operation and electrical energy costs.

\section{Materials and Methods}

In order to do so, a pilot test has been developed. The pilot test consists of three aging HPVs (high pressure vessels) and a test HPV. The aging HPV consists of three high pressure vessels where membranes of three main manufacturers have been essayed. The vessels are equipped with a control valve, a pressure transmitter and a flow meter in the permeate flow. Further instruments of the rack provided necessary information for the appropriate aging HPV monitoring.

The aim of the aging HPV was to achieve a mature performance of the membranes. During this aging process, 2 months, the main performance parameters have been controlled.

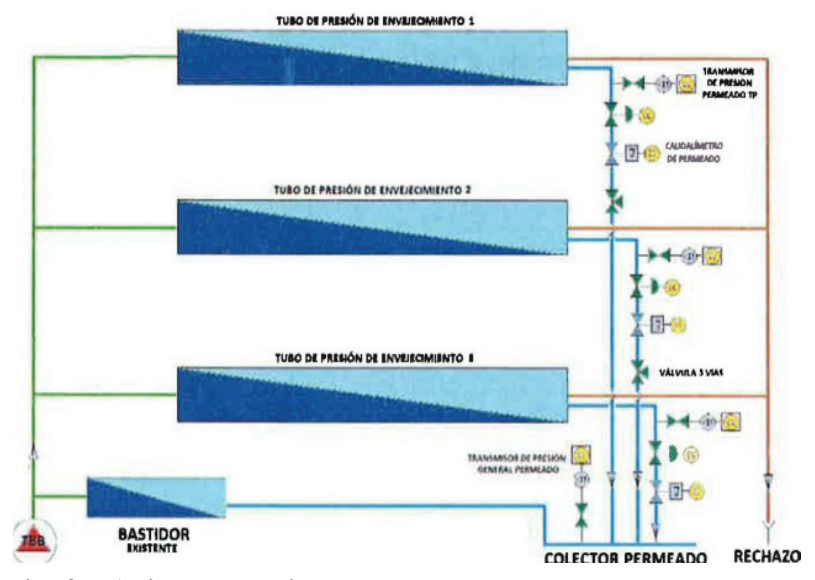

Fig. 2 Aging HPV diagram.

There are three pressure vessels in parallel with the other vessels of the train. The same feed flow and feed pressure are introduced to all three pressure vessels. There are three different types of reverse osmosis membranes, one model per each vessel.

We can test them in this way and it can be seen that the permeate flow of each aging pressure vessels is different, therefore we have to put the same feed pressure per each vessel so we use permeate pressure in each one in order to get it.

Due to this all three aging pressure vessels have installed a control valve for the Delta pressure, together a pressure transmitter, flow meter, control system and three vies valve, in the permeate pipe, seen in Fig. 2. This is under low pressure so it is not a big cost for the investigation.

We have to fix the same permeate flow per aging vessel and we can do it with a back pressure. In this way, the aging pressure vessel with the highest permeate pressure is the one with the lowest energy consumption. We need more back pressure in order to get the same permeate flow of the others. In this way we can get the conclusion of the more efficient type of reverse osmosis membranes. If we would have all the vessels of the trains with this type of membrane of the highest back pressure, we would be operating with higher feed pressure than now, saving energy costs, or producing more water at the same feed pressure than now.

Therefore, with this study, we know the most efficient reverse osmosis membrane, under these 
operating conditions of the plant.

The test HPV consists of a vessel equipped with all the required elements that leaded to a full monitoring and control of the operational conditions (pressure and flows of the seawater, brine and permeate flows).

The test HPV was equipped with:

- Feed seawater flow:

- A control valve at the HPV inlet for the water flow and pressure control;

- A pressure transmitter;

- A drop pressure transmitter.

- Permeate flow:

- A flow meter;

- A pressure transmitter (the one of the full scale rack).

- Brine flow:

- Three valves, in order to control the brine flow and appropriately make the brine discharge from 65 bars to atmospheric pressure;

\section{- A flowmeter.}

The feed flow was calculated by summing up brine and permeate flows.

The test HPV was designed in order to make it possible to test the three manufacturers' membranes under any possible condition, seen in Fig. 3. Any operation parameter (pressure or flow) could be modified by the control valves, and every main parameter (pressure, flows and water quality) could be measured.

The membranes tested to determine their performance under specifics conditions between different membranes manufacturers, at least during two weeks, have the same conditions (pressure, flow, temperature).

In the test pressure vessel we have also in the permeate pipe a flow meter, a pressure transmitter and a three vies valve, like in the aging vessels. Moreover, in this test vessel we have instruments in the brine pipe (three valves and a flow meter) and in the feed pipe (pressure transmitter, drop pressure transmitter and a control valve for the pressure and water flow control).

Under these conditions we can test the three different models of reverse osmosis membranes under real



Fig. 3 Test HPV diagram.

conditions, changing the feed pressure or feed flow, in order to get a permeate flow and also variating the recovery of the system. After this pilot test and with the information of the aging vessels, we can draw a different picture of the efficiency of each membrane in order to select the element with more energy saving.

Moreover, we have to take into account the permeate quality of each type of membrane, due to we have to produce water with TDS lower than $400 \mathrm{mg} / \mathrm{L}$ and Boron lower than $1 \mathrm{mg} / \mathrm{L}$ too. If all membranes are under these parameters, we can select the most efficient one which produces the nominal flow with lower energy consumption.

The whole process was monitored in a screen developed ad hoc in the SCADA of the full-scale plant. Therefore the process was followed $24 / 7$ by the plant operators.

In order to gather the appropriate information, the analytical program has been established as shown in Tables 1 and 2 .

The experiments have been carried out according to this schedule:

- Two months in a row in the aging HPV running the three HPV simultaneously. During this period, the membranes have been monitored and aged simultaneously and under the same conditions before being tested.

- Two weeks in a row in the test HPV. The membranes of each manufacturer have been loaded and tested in the test HPV in order to determine their performance under specific conditions. Results between different membranes manufacturers have been compared under the same experimental conditions (pressure, flow, temperature), as the experiments have been carried out alternatively in the test HPV. 
Table 1 Aging HPV analytical program.

\begin{tabular}{lllll}
\hline & & & \multicolumn{2}{l}{ Aging HPV analytical program } \\
\hline Parameter & Feed & Permeate & Brine & Analysis frequency \\
Water flow & $-*$ & $\checkmark$ & $-*$ & continuous (SCADA monitored) \\
$\begin{array}{l}\text { Pressure } \\
\text { Quality }\end{array}$ & $\checkmark$ (rack/HPV) & $\checkmark$ & $\checkmark$ & continuous (SCADA monitored) \\
SDI & $\checkmark$ & & & $8 \mathrm{~h}$ \\
Temperature & $\checkmark$ & $\checkmark$ & & daily (labor)/continuous (SCADA monitored) \\
Conductivity & $\checkmark$ & $\checkmark$ & $\checkmark$ & daily (labor)/continuous (SCADA monitored) \\
pH & $\checkmark$ & $\checkmark$ & $\checkmark$ & daily (labor)/continuous (SCADA monitored) \\
Boron & $\checkmark$ & - & & raw water: daily/permeate: weekly \\
Other (full scale plant analysis) & $\checkmark$ & $\checkmark$ & $\checkmark$ & monthly \\
\hline
\end{tabular}

* Only average feed/brine rack average values available.

Table 2 Test HPV analytical program.

\begin{tabular}{lllll}
\hline & & & \multicolumn{2}{l}{ Test HPV analytical program } \\
\hline Parameter & Feed & Permeate & Brine & Analysis frequency \\
Water flow & $\checkmark$ & $\checkmark$ & $\checkmark$ & continuous (SCADA monitored) \\
$\begin{array}{l}\text { Pressure } \\
\text { Quality }\end{array}$ & $\checkmark$ & $\checkmark$ & $\checkmark$ & continuous (SCADA monitored). $\Delta$ P available \\
SDI & & & & $8 \mathrm{~h}$ \\
Temperature & $\checkmark$ & & & daily (labor)/continuous (SCADA monitored) \\
Conductivity & $\checkmark$ & $\checkmark$ & $\checkmark$ & daily (labor)/continuous (SCADA monitored) \\
pH & $\checkmark$ & $\checkmark$ & $\checkmark$ & daily (labor)/continuous (SCADA monitored) \\
$\begin{array}{l}\text { Boron } \\
\text { Other (full scale plant analysis) }\end{array}$ & $\checkmark$ & $\checkmark$ & $\checkmark$ & raw water: daily/permeate: weekly \\
HPV probing & $\checkmark$ & $\checkmark$ & & $170 \mathrm{~h}$ (weekly) \\
\hline
\end{tabular}

The loop two months aging/two weeks testing periods have been repeated during the whole pilot.

A key aspect in the definition of the process was the hydraulic model and the election of the valves that would lead to an appropriate control of the system. Thus, a detailed study has been developed following the next steps:

(1) Environment definition: definition of the operational criteria and main parameters values under extreme testing conditions,

(2) Hydraulic study: according to the operational conditions needs a hydraulic study was developed.

In this study it has been designed the piping system. It has also been chosen the control valves in order to accomplish the control requirements of the pilot test based on the hydraulic model, Fig. 4.

\section{Conclusions}

The conclusions of this pilot test will lead to:

(1) Compare the membrane performance of the three main membrane manufacturers;

(2) To gather the required information to base future decision-making processes related to:

- Membrane replacement: in this case, the pre-existing high-pressure pump already determines the operation conditions (pressure and water flow). Nevertheless, it is possible to evaluate the optimal number of HPV and the new membrane configuration for the rack.

- Retrofit projects (with new membranes): under this perspective, the operational conditions may be redefined. It is possible to adapt the high-pressure pump/electrical motor to the new operational needs, under the new thermal scenario. There is also the possibility to introduce VFD (variable frequency drives) to adequate pressure to temperature. Therefore the study of the most efficient operational conditions for each membrane manufacturer and configuration is a key factor in order to design the most adequate. 




(a) Permeate of the aging HPV

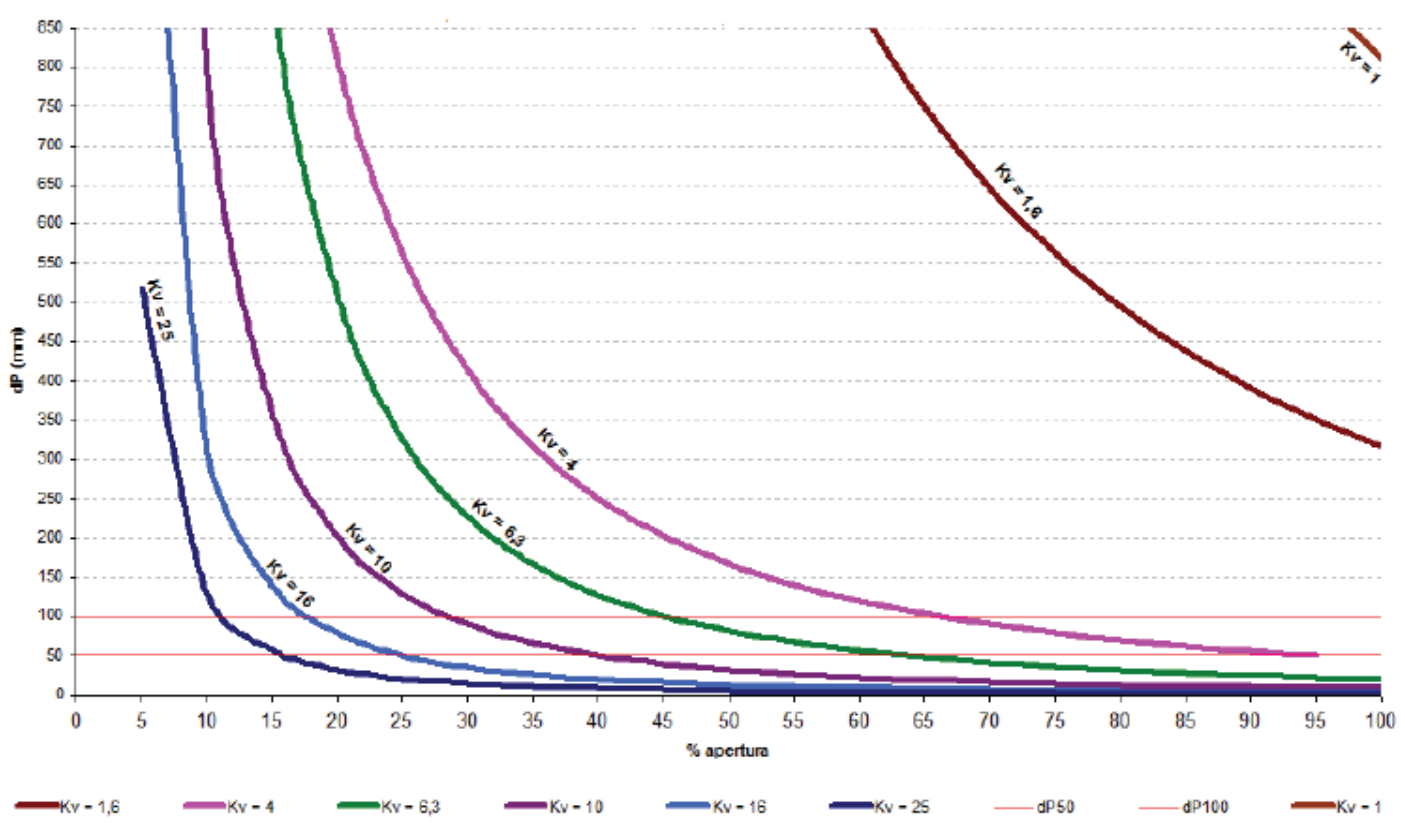

(b) Feed seawater flow of test HPV

Fig. 4 Comparative hydraulic model for control valve election.

In both cases (membrane replacement or retrofit projects), the pilot test may lead to reinforce the decision-making process with real data in order to choose the most efficient membrane and system configuration for the new thermal scenario, taking into account investment versus energetic efficiency in each case: membrane replacement or retrofit project.

Finally, as conclusions, we got to reduce the electrical consumption due to this high efficiency membrane test from $3.04 \mathrm{~kW} \cdot \mathrm{h} / \mathrm{m}^{3}$ with Pelton turbines to $2.60 \mathrm{~kW} \cdot \mathrm{h} / \mathrm{m}^{3}$ with ERI isobaric chambers. Moreover, we are thinking to reduce more the electrical 


\section{in Order to Reduce the Electrical Consumption}

consume introducing a Dweer device decreasing the electrical consumption up to $2.41 \mathrm{~kW} \cdot \mathrm{h} / \mathrm{m}^{3}$.

\section{References}

[1] Latorre, F. J. G., Báez, S. O. P., and Gotor, A. G. 2015. "Energy Performance of a Reverse Osmosis Desalination Plant Operating with Variable Pressure and Flow." Desalination 366 (June): 146-53.

[2] Gude, V. G. 2016. "Desalination and Sustainability-An Appraisal and Current Perspective." Water Research 89 (February): 87-106.

[3] Schallenberg-Rodriguez, J., Veza, J. M., and Blanco-Marigorta, A. 2014. "Energy Efficiency and Desalination in the Canary Islands." Renewable and Sustainable Energy Reviews 40 (December): 741-8.

[4] Mazlan, N. M., Peshev, D., and Livingston, A. G. 2016.
"Energy Consumption for Desalination-A Comparison of Forward Osmosis with Reverse Osmosis, and the Potential for Perfect Membranes.” Desalination 377 (January): 138-51.

[5] Song, D., Wang, Y., Xu, S., Gao, J., Ren, Y., and Wang, S. 2015. "Analysis, Experiment and Application of a Power-Saving Actuator Applied in the Piston Type Energy Recovery Device.” Desalination 361 (April): 65-71.

[6] Dow, N., and Gray, S. et al. 2016. "Pilot Trial of Membrane Distillation Driven by Low Grade Waste Heat: Membrane Fouling and Energy Assessment." Desalination 391 (August): 30-42.

[7] Zhao, R., Porada, R. et al. 2013. "Energy Consumption in Membrane Capacitive Deionization for Different Water Recoveries and Flow Rates and Comparison with Reverse Osmosis.” Desalination 330 (December): $35-41$. 